\title{
Intramedullary cervical spinal mass after stem cell transplantation using an olfactory mucosal cell autograft
}

\author{
Claire F. Woodworth MA MD, Gregory Jenkins BSc MD, Jane Barron MSc MD, Nanette Hache BMedSci MD
}

- Cite as: CMAJ 2019 July 8;191:E761-4. doi: 10.1503/cmaj.181696

A

38-year-old man was referred to the neurosurgical clinic with a 3- to 4-year history of deteriorating neurologic function in his arms (sensory disturbance with some subjective weakness) and gradual loss of bladder function.

The patient's medical history included a $\mathrm{C} 5 / \mathrm{C} 6$ fracturedislocation secondary to trauma at 20 years of age. Despite having had posterior spinal fusion and rehabilitation, he had partial paralysis of his arms, and severe paralysis of his legs and trunk. According to the American Spinal Injury Association (ASIA) Impairment Scale (AIS) (https://asia-spinalinjury.org/wp-content /uploads/2019/04/ASIA-ISCOS-IntIWorksheet_2019.pdf), which grades impairment after spinal cord injury from A (complete motor and sensory deficits) to $E$ (neurologically intact), he had sustained an ASIA D injury (i.e., motor incomplete). This means that half or more of the key muscles below the neurologic level of injury were active against gravitational resistance. At 26 years of age, the patient had undergone experimental stem cell transplantation. Using a nasal approach, the surgical team harvested an olfactory mucosa graft that was transplanted to his spinal cord lesion on the next day. Although the patient could not provide an operative report, the surgical team (Lima and colleagues) published their transplantation and surgical procedure. ${ }^{1}$ Fusion hardware was removed and he again underwent rehabilitation. Although he hoped to walk again and have resolution of his chronic pain, he never regained clinically significant motor function, and he experienced additional pain.

When the patient presented to us with deteriorating neurologic function, initial investigations included magnetic resonance imaging (MRI), which showed edema within the cervical cord and a discrete intramedullary lesion extending from about C4-C7 (Figure 1). The preoperative differential diagnosis included mass related to the stem cell transplant, ependymoma, astrocytoma and, less likely, hemangioblastoma.

The patient was brought to the operating room for posterolateral decompression of $\mathrm{C} 4-\mathrm{C} 7$ bilaterally and resection of the intramedullary spinal cord mass. We identified a large mass with several compartments. There was considerable mucinous material that was very thin and could be expressed (Figure 2). There were also many solid components that were adherent

\section{KEY POINTS}

- Although there is legitimate research being done on stem cell therapies, a large industry targets patients in developed countries with unproven treatments, using Internet-based marketing.

- Mass lesion is a delayed complication of stem cell transplant.

- There is low-quality evidence for olfactory mucosal autograft surgery in patients with spinal cord injury.

- Physicians in Canada should be aware of stem cell tourism because they may need to counsel patients on transplantation or diagnose complications in those who have had such procedures.

and infiltrative to the spinal cord. We completed a subtotal resection and took multiple samples.

Pathologic examination of the samples found mucinous material and tissue consistent with ectopic olfactory mucosa. The sections showed tissue and small cysts lined by a pseudostratified columnar epithelium (Figure $3 \mathrm{~A}$ ). Nerve twigs and submucosa glands were seen in the underlying connective tissue, with 1 slide showing bone. Results from examination of tissue using immunohistochemistry showed neurofilaments and S100 proteins within the nerve twigs, and perineurial cells that were positive for epithelial membrane antigen and glial fibrillary acidic protein positivity in some areas, likely representing gliotic spinal tissue (Figure 3B).

Between discharge and follow-up at 6 weeks, the patient became severely deconditioned because of admissions to hospital for urinary tract infection and acute pancreatitis. His injury remained classified as ASIA D, but a physical examination found either stability or slight loss of power in the myotomes of his arms and legs. Given the subtotal resection of the mass, radiotherapy was offered after consultation with colleagues in other centres in Canada to try to sterilize any residual cells in the hope of slowing regrowth of the mass. The benefit of radiation in this setting is unknown; however, further surgical resection was not possible, and there was no role for chemotherapy. Treatment with radiotherapy and rehabilitation and surveillance are ongoing. 


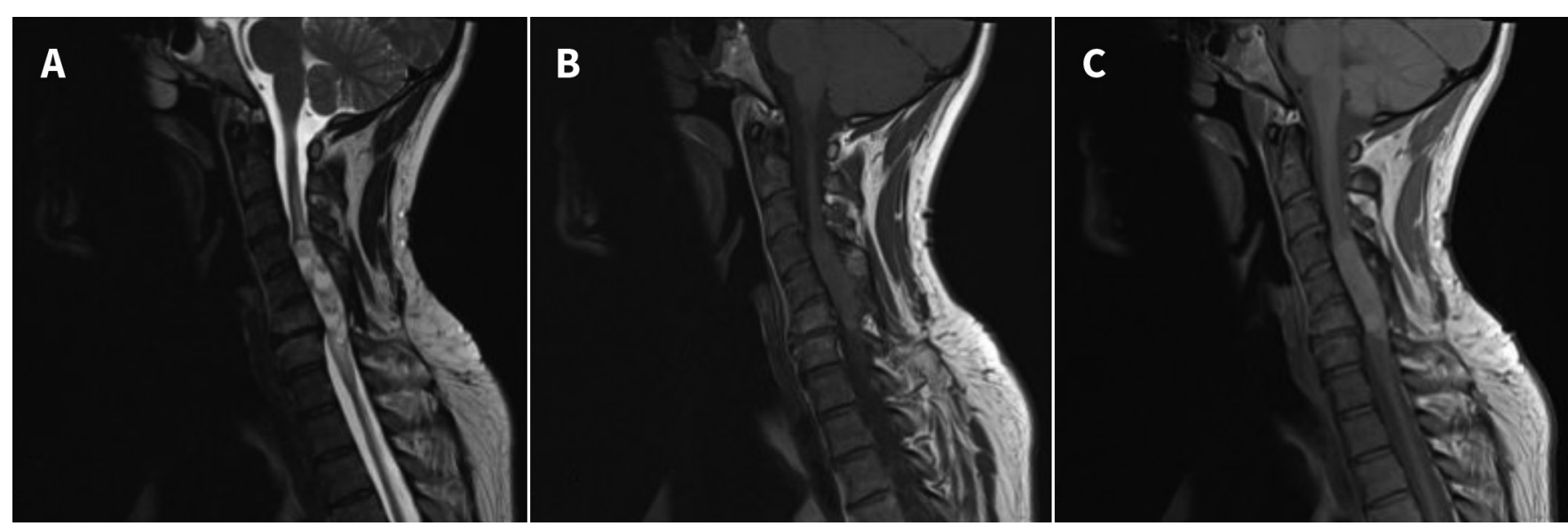

Figure 1: Sagittal (A) $T_{2}$-weighted, (B) $T_{1}$-weighted and (C) proton density magnetic resonance images of an intramedullary cervical spinal mass in a 38-year-old man 12 years after olfactory mucosal autograft surgery. A discrete expansile lesion extends from about $\mathrm{C} 4$ to $\mathrm{C} 7$ (maximal dimensions of $\left.5.5 \times 1.3 \mathrm{~cm}^{2}\right)$. The images in (A) and (C) show a multiloculated with heterogeneous intermediate to high signal intensity on the $T_{2}$-weighted and proton density images. High signal intensity within the cord above and below the lesion in (B) suggests edema. We did not administer gadolinium because the patient had reduced renal function.

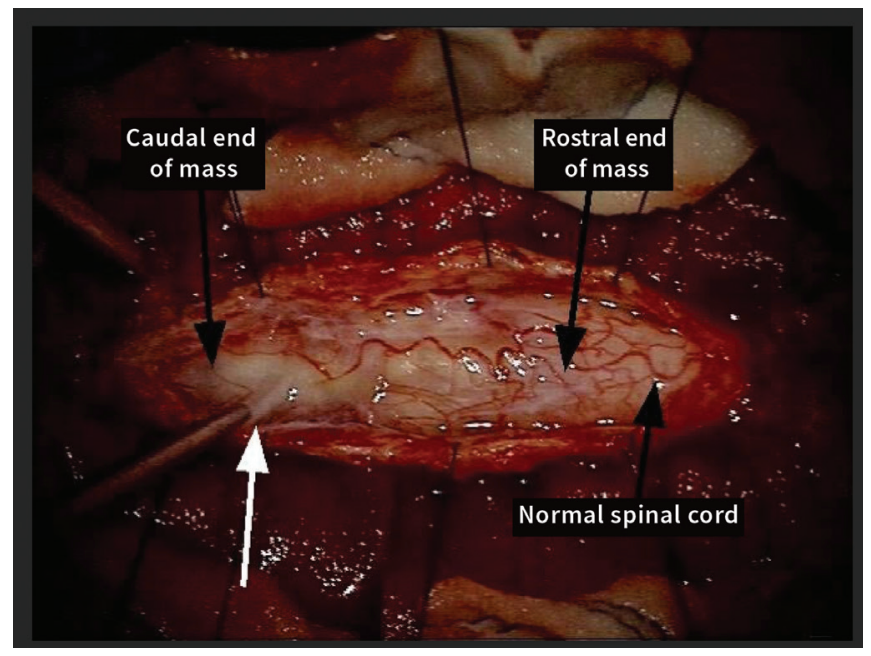

Figure 2: Intraoperative view of the intramedullary spinal cord mass. The suction catheter shows very thin mucinous material that was expressed from the mass (white arrow). Solid components were adherent and infiltrative to the spinal cord.

\section{Discussion}

Even though legitimate research is being done on stem cell therapies, an international industry has developed that sells unproven cosmetic and medical stem cell-based treatments, with the United States having the largest number of stem cell clinics globally. ${ }^{2}$ Individuals in developed economies are engaging in "stem cell tourism," despite limited regulation and emerging reports of adverse events. A 2018 analysis of stem cell-based treatments found 35 cases describing acute or chronic complications or death. ${ }^{3}$ Postintervention complications were neoplastic, infectious, neurologic (e.g., loss of vision after intravitreal injections) and cardiovascular (e.g., ventricular fibrillation after myocardial injection or pulmonary embolus). Although some of the reported adverse events might relate to surgical technique alone, others are likely the direct result of the yet unproven treatments using stem cells.
Autologous stem cell treatment is theorized to improve functional outcomes through several methods. ${ }^{4}$ One proposed mechanism is that the presence of stem cells provides neuroprotection through mitigation of the normal posttraumatic inflammatory response, which reduces the amount of delayed cell loss. Transplanted cells are thought to express bioactive molecules, such as cytokines and trophic factors, stimulating local gliosis and angiogenesis. Stem cells may also promote axon regeneration and plasticity by forming bridges or alternate relays across a spinal cord lesion.

The surgical technique for stem cell transplantation involves harvesting and preparing the stem cells, as well as modifying scar tissue or cystic changes at the graft site before implantation. ${ }^{1}$ Postoperative rehabilitation seemingly enhances stem cell treatment (reference 1, Appendix 1, available at www.cmaj.ca/ lookup/suppl/doi:10.1503/cmaj.181696/-/DC1), although the most optimal type and duration remain unknown.

Investigated cell types include Schwann cells, olfactory ensheathing cells, mesenchymal stem cells, neural stem and progenitor cells, and embryonic cell-derived or inducible pluripotent stem cell-derived neural precursor cells. ${ }^{5}$ In our patient, olfactory mucosa was used as an autologous graft because it is readily accessible and contains neural stem and progenitor cells and olfactory ensheathing cells. ${ }^{1}$ It is postulated that these cells maintain a lifelong capacity to differentiate into neurons and glia while also supporting axonal regrowth. ${ }^{4}$

\section{Olfactory mucosal autografts}

An emerging body of work lends some credence to the efficacy of olfactory mucosal autograft for recovery from spinal cord injury. Animal models have shown partial locomotor recovery after transplantation of olfactory mucosa into transected rat spinal cords. ${ }^{6}$ Lima and colleagues published a prospective, uncontrolled pilot study of olfactory mucosal autograft implantation in 20 patients who had sustained traumatic spinal cord injury 18 to 189 months previously (mean 49 months). ${ }^{7}$ The AIS scores improved in 11 of 20 patients and declined only in 1 patient. Voluntary electromyography was restored below the level of injury 


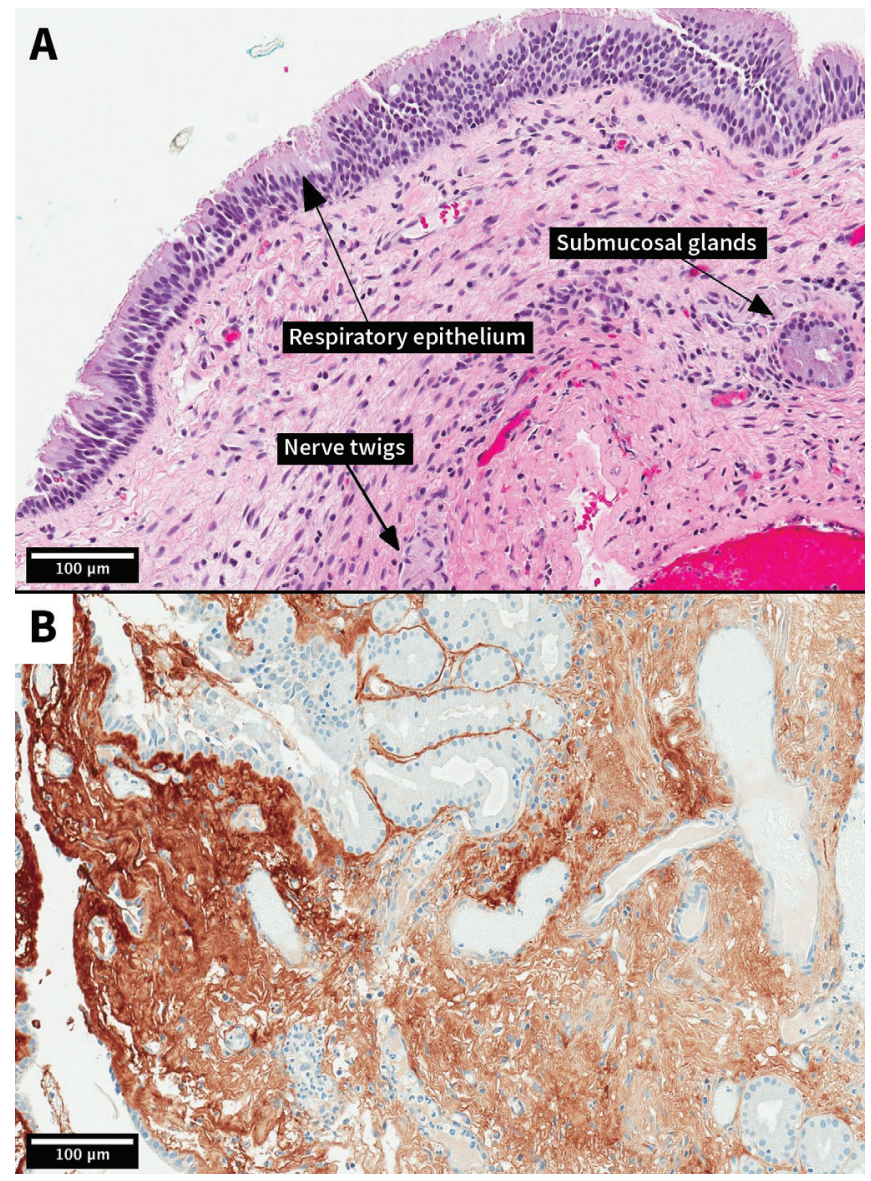

Figure 3: Photomicrographs showing the olfactory mucosa autograft (original magnification, $\times 200$ ). (A) Spinal cord mass (hematoxylin and eosin stain). Respiratory epithelium is visible with underlying submucosal connective tissue. In the epithelium, ciliated cells predominate, but some goblet cells are also present. There are nerve twigs and glands in the submucosa. (B) Gliotic spinal cord tissue (brown) adjacent to respiratory mucosal tissue (glial fibrillary acidic protein stain).

in 15 patients, whereas 4 patients had new somatosensory evoked potentials, supporting the conductivity of olfactory mucosa autografts. Likewise, a case report in 2016 found elicited motor evoked potentials in a patient with a long-term ASIA A spinal cord injury, which suggests central nervous system conductivity along corticospinal pathways after olfactory mucosal autograft transplantation. ${ }^{8}$ Other small studies utilized these outcome measures to report similar findings (reference 2, Appendix 1).

Studies involving transplantation using olfactory mucosal autografts notably carry several limitations. To date, patient enrollment is small, making the generalizability of results difficult. The studies are neither randomized nor blinded. The relative contributions of intensive postoperative rehabilitation and olfactory mucosa transplantation to recovery are unclear given the lack of large control groups. Some studies with questionable rehabilitation intensity and compliance showed no postoperative improvement in ASIA motor score. ${ }^{7,9}$ Although these studies claim that olfactory mucosal autograft transplantation is feasible and safe given the few adverse events encountered, their followup periods are limited. For example, Lima and colleagues followed patients for a mean duration of 27.7 months (range of 12-45 months), during which MRI showed no mass or syringomyelia at the lesion sites. ${ }^{7}$ Autograft mass lesions appear to have a longer development time.

There have been several other cases of mass lesions following olfactory mucosal autograft reported in the literature..$^{10,11}$ Dlouhy and colleagues described an autograft-derived spinal cord mass with the same appearance as the mass in our patient. ${ }^{10}$ An 18-year-old woman sustained a traumatic T10-11 ASIA A spinal cord injury and underwent olfactory mucosal cell transplantation with the same medical team as our patient. About 8 years later, she developed a heterogeneous, multicystic, mucinous mass. On pathologic examination, this mass was composed of respiratory epithelium, submucosal glands with goblet cells and intervening nerve twigs, as in our case. She also did not have any neurologic benefit in the interim. Two other cases involving patients who developed similar spinal masses 5 and 7 years after autologous olfactory mucosa transplantation have been reported. ${ }^{11}$

It is difficult to determine the true rate of mass lesions in patients with olfactory mucosa autografts. Of the more than 140 patients (reference 3, Appendix 1) who had an autologous olfactory mucosal autograft by Lima and colleagues, our patient had the second reported spinal mass. ${ }^{10}$ Given the latency period between transplantation and presentation in our patient, it is possible that new masses will be diagnosed in others who have already had stem cell transplantation. Notably, there are additional case reports of masses after stem cell tourism for other conditions (references 4-6, Appendix 1).

Olfactory mucosal autograft surgery costs about $\$ 50000$; with patients facing additional expenses for travel, accommodations, caregiver support and recuperation, this price can easily double. The subsequent cost for managing any complications in Canada can be the same because these patients require ongoing multidisciplinary care.

There have been calls for physicians, medical societies and licensing boards to recognize the evolving body of legitimate stem cell research while simultaneously familiarizing themselves with stem cell tourism in an effort to prevent patient morbidity and mortality. ${ }^{12}$ Health Canada has recently issued a position paper (www.canada.ca/en/health-canada/services/drugs -health-products/biologics-radiopharmaceuticals-genetic-therapies /applications-submissions/guidance-documents/cell-therapy-policy .html) clarifying that autologous cell therapy products are considered drugs. As such, they are subject to regulation and require authorization for use.

\section{Conclusion}

Given the vulnerability of patients who are chronically ill, especially those with spinal cord injury or neurologic disorders who may be targets of Internet-based marketing for stem cell therapy), physicians in Canada should be aware of the rationale behind stem cell therapy as well as the reported adverse events. Both family physicians and specialists may need to counsel patients on stem cell transplantation or diagnose complications in those who have had these procedures. 


\section{References}

1. Lima C, Pratas-Vital J, Escada P, et al. Olfactory mucosa autografts in human spinal cord injury: a pilot clinical study. J Spinal Cord Med 2006;29:191-203, discussion 204-6.

2. Berger I, Ahmad A, Bansal A, et al. Global distribution of businesses marketing stem cell-based interventions. Cell Stem Cell 2016;19:158-62.

3. Bauer G, Elsallab M, Abou-El-Enein M. Concise review: a comprehensive analysis of reported adverse events in patients receiving unproven stem cell-based interventions. Stem Cells Transl Med 2018;7:676-85.

4. Assinck $P$, Duncan GJ, Hilton BJ, et al. Cell transplantation therapy for spinal cord injury. Nat Neurosci 2017;20:637-47.

5. Chhabra HS, Sarda K. Clinical translation of stem cell based interventions for spinal cord injury - Are we there yet? Adv Drug Deliv Rev 2017;120:41-9.

6. Lu J, Féron F, Mackay-Sim A, et al. Olfactory ensheathing cells promote locomotor recovery after delayed transplantation into transected spinal cord. Brain 2002;125:14-21.

7. Lima C, Escada P, Pratas-Vital J, et al. Olfactory mucosa autografts and rehabilitation for chronic traumatic spinal cord injury. Neurorehabil Neural Repair 2010;24:10-22.

8. Iwatsuki K, Tajima F, Sankai Y, et al. Motor evoked potential and voluntary EMG activity after olfactory mucosal autograft transplantation in a case of chronic, complete spinal cord injury: case report. Spinal Cord Ser Cases 2016;2: 15018.

9. Chhabra HS, Lima C, Sachdeva S, et al. Autologous olfactory [corrected] mucosal transplant in chronic spinal cord injury: an Indian Pilot Study [published erratum in Spinal Cord 2009;47:904]. Spinal Cord 2009;47:887-95.

10. Dlouhy BJ, Awe O, Rao RC, et al. Autograft-derived spinal cord mass following olfactory mucosal cell transplantation in a spinal cord injury patient: case report. J Neurosurg Spine 2014;21:618-22.

11. Chen KS, McIntyre JC, Lieberman AP, et al. Human spinal autografts of olfactory epithelial stem cells recapitulate donor site histology, maintaining proliferative and differentiation capacity many years after transplantation. Acta Neuropathol 2016;131:639-40.

12. Bowman M, Racke M, Kissel J, et al. Responsibilities of health care professionals in counseling and educating patients with incurable neurological disease regarding "stem cell tourism": caveat emptor. JAMA Neurol 2015;72:1342-5.
Competing interests: None declared.

This article has been peer reviewed.

The authors have obtained patient consent.

Affiliations: Departments of Radiology (Woodworth, Hache), Neurosurgery (Jenkins) and Pathology (Barron), Faculty of Medicine, Memorial University of Newfoundland, St. John's, Nfld.

Contributors: All of the authors were involved in conceiving and drafting the manuscript, revised it critically for important intellectual content, gave final approval of the version to be published and agreed to be accountable for all aspects of the work.

Acknowledgements: The authors thank Dr. Roger Avery for his contributions to patient care and his retrieval of the intraoperative photograph. The authors also thank Dr. Peter Bartlett for his contributions to the intellectual content of this manuscript.

Correspondence to: Nanette Hache, nhache@yahoo.com

The section Cases presents brief case reports that convey clear, practical lessons. Preference is given to common presentations of important rare conditions, and important unusual presentations of common problems. Articles start with a case presentation (500 words maximum), and a discussion of the underlying condition follows (1000 words maximum). Visual elements (e.g., tables of the differential diagnosis, clinical features or diagnostic approach) are encouraged. Consent from patients for publication of their story is a necessity. See information for authors at www.cmaj.ca. 